\title{
A Critical Evaluation of RFID in Manufacturing
}

\author{
Wei Zhou ${ }^{1,3}$ and Selwyn Piramuthu ${ }^{2,3}$ \\ 1 Information \& Operations Management, ESCP Europe, Paris, France \\ ${ }^{2}$ Information Systems and Operations Management, University of Florida \\ Gainesville, Florida 32611-7169, USA \\ 3 RFID European Lab, Paris, France \\ wzhou@escpeurope.eu, selwyn@ufl.edu
}

\begin{abstract}
The number and extent of supply chain-related RFID applications far exceed those in the general manufacturing domain. We discuss possible reasons for this state. We review existing applications of RFID in manufacturing and discuss possible applications and related advantages and challenges.
\end{abstract}

\section{Introduction}

Since the early 2000s, there has been a tremendous increase in applications involving RFID (Radio-Frequency IDentification) tags. A majority of these applications have been somehow associated with supply chains or pharmaceutical industry or both. Major reasons for this include the huge impetus from WalMart and the U.S. Department of Defense (DoD) as well as various ePedigree and anti-counterfeit initiatives from large pharmaceutical firms. With the successful incorporation of RFID tags on pallets or even individual items that pass through supply chains, there has been increasing interest among stake-holders from other application areas (e.g., tickets used in public transportation, library books). While there is some spill-over effect, the general manufacturing domain has not received its fair share of attention for RFID implementations. The same is true for RFID-related research publications in the manufacturing domain.

RFID tags have several characteristics in a desirable form factor that allows for its dominance over other existing auto-identification technologies. These tags, with minimal memory and processing power, have unique identifiers and are capable of carrying on extended conversation with a reader. This capability facilitates unique identification of any given tag among a set of tags on a large scale as well as storing and retrieving essential information about the tagged object. Other beneficial characteristics of RFID tags include their readability without direct line-of-sight (unlike bar codes) whereby they can be embedded inside objects and still be read, their durability in harsh environments, and the ability to be (re-)programmed on-the-fly. These characteristics certainly render RFID tags their attractiveness in manufacturing environments where their presence can facilitate mass customization. Overall, the ability to identify and store/update information about an object at item-level and to communicate this information on demand enables its seamless integration in an automated system

C. Emmanouilidis, M. Taisch, D. Kiritsis (Eds.): APMS 2012, Part II, IFIP AICT 398, pp. 41-48, 2013.

(C) IFIP International Federation for Information Processing 2013 
(Zhou 2009). While progress in bar code technology allows for the possibility to store more information using two-dimensional bar codes, these can't be updated nor do they have the ability to carry on a two-way conversation.

For example, item-level information can readily be used to match the most appropriate set of components that go into the final product (e.g., Zhou and Piramuthu, 2012). This would reduce the amount of wastage due to final products that are beyond pre-specified tolerance levels, while also improving the quality of the final products resulting from reduced mismatch-related issues. Process mining of the paths taken by RFID-embedded pallets in a manufacturing shop floor can also be used to develop a map for appropriate placement of machines to improve the efficiency of the entire system (e.g., Hu et al. 2012). Sensorenabled RFID tags mounted on the component or on the substrate carrying the component can be used to ensure that this component was processed properly, for example, in terms of appropriate temperature range and the amount of time spent at any given temperature (e.g., Zhou and Piramuthu 2011). The underlying thread among these applications is that item-level RFID tags greatly facilitate manufacturing with real-time information (e.g., Hua et al. 2008).

Increasingly, RFID tags are being introduced in more and more manufacturing environments. For example, in Ford's Essex Engine plant at Windsor Ontario, the entire work sequence for an engine is stored in its RFID tag when it starts down the line (Sharp 1999). As it reaches different stations, it is interrogated to determine the tasks that require completion. Moreover, results from (e.g., quality check) tests are stored locally in the tag. In Ford's Cuautitlan Mexico facility that produces 300-400 thousand cars and trucks per year, apparently they were previously using a manual paper-based identification system to keep track of production flows and vehicles. This manual system unfortunately was ineffective due to frequent errors and costs associated with production oversights. Moreover, identification sheets that were used to track the vehicles frequently suffered loss and were oftentimes switched or damaged, rendering it difficult to control manufacturing quality. The introduction of RFID-based system addressed several of these issues ( $\mathrm{Li}$ et al. 2004). For building Passat cars at the Emden plant, Volkswagen tags 20,000 parts each day to efficiently utilize its press shop's two large $73,000 \mathrm{KN}$ presses that require a smooth material flow system and very high machine availability downstream. Sheets cut from coils are placed on pallets that are RFID-tagged. These tags contain quality and product data at an early stage in the process to ensure the traceability of each pressed part. This also enables transparency in the material flow and for the material flow to match production demand. Audi incorporated semi-passive tags that can withstand high temperatures and painting into the assembly process of its TT sports cars at its Ingolstadt manufacturing plant. Assembly instructions and other information such as the chassis' intended color and other process information are stored in these reusable tags for use by robots at about 80 manufacturing and assembly stations. These tags are designed to ensure that appropriate steps occur at the right time and to the right chassis and to help control the flow of the chassis through the production line. When a defect is discovered, the system allows for 
flexibility in changing the production sequence. As a part of the RFID-based Automotive Network (RAN) project, a consortium of about 20 automobile manufacturers, suppliers, logistics firms, research institutes and software companies is testing the use of RFID in production and logistics processes within Germany's automotive industry. Daimler uses RFID to track production containers between Stuttgart, Berlin and their plant in Tuscaloosa, Alabama. BMW monitors containers as they move between their facilities and suppliers along the production chain. Opel tests the continuous use of RFID from vehicle manufacturing and the entire supply chain until its use by the consumers.

Clearly, several initiatives with RFID in manufacturing as the focus have begun not only in automotive manufacturing but also in a wide variety of other manufacturing facilities. We briefly discuss related literature in the next section. In the following section, we critically evaluate the characteristics, advantages, as well as issues related to the incorporation of RFID tags in a manufacturing context. We conclude the paper with a brief discussion.

\section{Literature Review}

Given the structural similarities and natural synergy between item-level information and agent-based systems, several studies have combined these in developing their manufacturing system frameworks. For example, Wang et al. (2009) develop an agent-based agile manufacturing planning and control system (AMPCS) to respond to the dynamically changing manufacturing activities and exceptions. AMPCS comprises modules for controlling the manufacturing system, generating production and operations schedule through an agent-based advanced manufacturing planning, and for performance analysis. Using AMPCS, they show that the agile manufacturing planning and control system developed through the integration of RFID and multi-agent system enables visibility, accountability, track-ability, responsiveness, and flexibility in a distributed and dynamic manufacturing system. Zhang et al. (2011) develop a Smart Gateway technology that captures real-time production data through different types of RFID/Auto-ID devices. Each such tagged entity forms a smart object (SO). Using Web services, they develop and manage agents based on a service-oriented architecture (SOA). They illustrate their framework using an assembly workstation where a Smart Gateway is deployed in real-time for defining, configuring and executing assembly operations. Trappey et al. (2009) develop agent-based collaborative mold production (ACMP) that supports the collaborative and autonomous mold manufacturing out-sourcing processes. ACMP provides autonomous features to handle vendor selection, task selection, and track in real-time the progress of outsourced tasks. They use Analytic Hierarchy Process (AHP) to solve the vendor selection and task selection problems and use RFID to provide real-time tracking capability for remote collaboration, control and monitoring out-sourcing partners. Higuera et al. (2007) develop an RFID-enhanced information management system (RFID-IMS) and illustrate how a multi-agent system model can be used for controlling a machining system incorporating RFID-IMS technology. 
Other studies consider RFID systems from different perspectives. We briefly discuss a select few. For example, Ivantysynova et al. (2009) observe the lack of dedicated models for determining the intangible and non-quantifiable costs and benefits of RFID adoption decisions in the manufacturing domain. They then suggest some guidelines to assess both the quantifiable and the non-quantifiable aspects of RFID in manufacturing. Using insights gained from case studies of production plants in different industries, they present a structured model that help guide decision makers understand the important trade-offs that need to be considered in the manufacturing domain.

Thiesse and Fleisch (2008) observe that the use of real-time location systems (RTLS) technology including RFID provides the opportunity for new levels of process visibility and control in comparison to conventional material-tracking systems. Specifically, they introduce dispatching heuristics that include the location information of each entity that waits to be processed in the manufacturing shop floor. They then illustrate the performance of their heuristics using an example scenario from a semiconductor manufacturing environment. Their results indicate that incorporating location information in dispatching heuristics helps accelerate existing processes and leads to efficiency gain.

Huang et al. (2008) show improvements in the effectiveness of managerial decisions and operational efficiency through real-time visibility provided by RFID tags. They argue that this level of visibility closes the loop of adaptive assembly planning and control. They propose and illustrate a high-level assembly line explorer facility that the line manager can use to oversee the status of the entire assembly line and a lower-level workstation explorer facility for operators to monitor the status of their operations at corresponding workstations. Through information generated at these different levels of granularity, they show that appropriate levels of control can be implemented at both local and global levels.

Zhou and Piramuthu (2011) study the process of remanufacturing through RFID-generated item-level information. Specifically, they consider the state of a component before it is remanufactured and attempt to match that component with its most appropriate complement.

Hozak and Collier (2008) use a simulation model to illustrate how RFID facilitates increased traceability and control in manufacturing, which in turn enables the use of more lot splitting and smaller lot sizes. They provide insights on operating policies (RFID vs. bar-code tracking mechanisms, extent of lot splitting, and dispatching rules) and an operating condition (setup to processing time ratio) that affect the mean flow time and proportion of jobs tardy in a job shop. Their results show that (i) performance worsens when bar code is used in the presence of extensive lot splitting, (ii) process changes such as extensive lot splitting may be necessary to justify RFID, (iii) the earliest operation due date dispatching heuristic is attractive under extensive lot splitting conditions, and (iv) the performance improvement with RFID under conditions of increased lot splitting is more when the setup to processing time ratio is small.

Brintrup et al. (2010) use the seven Toyota Production System wastes as a template and view RFID technology as a vehicle to achieve leaner 
manufacturing through automated data collection, assurance of data dependencies, and improvements in production and inventory visibility. They test their tool-set on two cases from push-based multi-national fast moving consumer goods manufacturing companies. Their results, developed through opportunity analysis, illustrate the identification of areas for improvement and other areas of value.

$\mathrm{Hu}$ et al. (2011) use RFID-generated path data in a manufacturing shop floor to develop their MP-Mine algorithm based on the Apriori association rule mining algorithm. Their goal is to develop a means to predict object movement patterns by mining frequent path information. Their results indicate the effectiveness of their algorithm, which can be used to predict the next location and arrival time of each entity in a manufacturing shop floor. In a similar vein, Arkan and Landeghem (2011) place RFID tags on the floor or on Automated Guided Vehicles (AGVs) in a manufacturing shop floor environment and study their movements, through readers placed on the ceiling, and then use this information to determine appropriate placement location for the processing machines.

While a majority of studies on RFID in manufacturing do not differentiate items that are tagged from those that are not, Li et al. (2004) argue otherwise. Sometimes, not all components that are manufactured need to be tagged. Li et al. (2004) argue that only a selected set of smart parts need to be tagged and that even these parts are not tagged during their entire stay in a manufacturing shop floor. The criticality of observing detailed conditions of a smart part at certain locations in a shop floor dictates what is tagged and for how long. They discuss a system that deals with smart parts as one that first identifies parts that need to be 'smart' (through RFID tags) at any point in time and a system that allows for seamless communication between such smart parts and equipment used in automated flexible manufacturing environment that also includes quality control, packing, storage and delivery.

\section{$3 \quad$ RFID in Manufacturing}

As discussed earlier, RFID's characteristic that is useful the most in a manufacturing context is its ability to store, modify, and communicate item-level information. As customers demand customized products, there is an increasing trend toward mass customization of manufactured products. A traditional manufacturing system does not easily lend itself to customization since its effectiveness derives from its ability to efficiently produce a large number of similar products. A majority of existing mass customization initiatives involve human(s) in the loop and this invariably results in slowing the process as well as introducing an appreciable amount of errors. It is difficult to automate a manufacturing system when mass customization is desired. However, the incorporation of RFID in manufacturing shop floors help alleviate this issue, as discussed earlier with examples from the automobile industry.

While RFID tags are rather flexible in their use, several issues still remain. The issues discussed in Kapoor et al. (2009) as related to supply chains are 
also relevant to RFID use in manufacturing environments. Moreover, a manufacturing environment has its own idiosyncratic characteristics that pose some additional challenges for RFID use. For example, when items pass through a supply chain, they are generally not processed along the way whereas this is not true in a manufacturing environment. When an RFID-tagged item goes through processing (e.g., milling, heat-treatment), at least two issues need to be addressed: (1) where (i.e., the physical location) to place the RFID tags on each item, and (2) the durability of these tags under extreme (e.g., temperature, pressure) conditions. For example, how and where does one place an RFID tag on a steel sheet that is pressed to form another shape in a high-pressure press and then heat-treated at high temperatures?

Several studies (e.g., Arkan and Landeghem 2011) consider the movement of entities as they are processed in a manufacturing shop floor environment. They generate a map of these movements either through placement of RFID tags on AGVs with readers on the ceiling or on (or slightly below the surface of) the floor with RFID readers on the AGVs and then mining the data thus generated for patterns. They then use these patterns to determine the most appropriate distribution of machines on the manufacturing shop floor. When RFID tags are placed on (or slightly under) the floor, two issues need to be considered: (1) use of physically stronger RFID tags (e.g., those that are embedded in a hard to break plastic or resin strip) and (2) readability of these tags, since it is difficult to read tags that are closer to steel reinforcements in the floor. This is another issue with the use of RFID tags in a manufacturing environment - the presence of materials (e.g., liquid, metal) that render it difficult to read RFID tags. Although progress is being made to alleviate this issue (e.g., through placement of a 'buffer' between the tag and the substrate metal or the use of an appropriate communication frequency).

In addition to process mining, it has been shown that traditional dispatching heuristics can be improved with the incorporation of part location information (e.g., Thiesse and Fleisch 2008). While this is a good first step, it is also possible to extend this further by learning the best (distance-based and otherwise) heuristic to use based on an instantaneous snapshot of the manufacturing shop floor (since any given dispatching heuristic is not dominant across different manufacturing shop floor conditions). Learning the best dispatching heuristic to use for any given set of instantaneous pattern exhibited by the shop floor and then instantiating them at appropriate time instances is readily accomplished using a knowledge-based system (e.g., Kriett et al. 2012).

In automobile manufacturing assembly lines, reusable RFID tags are generally placed on the side of a skid carrying the chassis down the production line via a conveyor system. The specific details (e.g., color, type of engine, other options) are stored in the RFID tag, which is then read and updated along the way as the process of manufacturing/assembly proceeds. Upon completion of work on a given chassis, the information stored in the RFID tag on the skid is refreshed with the specifications of the next chassis it carries. While this certainly facilitates the manufacturing/assembly process, RFID tag(s) attached to the automobile 
with necessary information would aid in maintenance and repair when it's with its owner (i.e., customer) in the long term.

Tolerance is important in manufacturing where consistencies in small levels of tolerance are difficult to achieve with limited resources. This results in discarded items when they fall even slightly outside the pre-specified tolerance limit. While this is an issue when only one item is considered, the extent of the problem explodes with the number of parts that are to be put together as a final product increases with each of these parts having their own tolerance limits (Zhou 2009, Zhou and Piramuthu 2012). For example, the best case scenario that illustrates the power of item-level information is a final product with two components - one that is at the positive extreme of its tolerance limit and the other at the negative extreme of its tolerance limit - that is close to a perfect match even though each of these are close to being discarded. Using item-level tolerance information stored in RFID tags, the different components can be matched appropriately resulting in a good (if not optimal) quality final product.

\section{Discussion}

RFID is a promising technology for manufacturing environments where automation is the norm. Through automation of storing, modifying, and retrieving critical information on parts that are processed in these environments, common errors due to mis-specification (when manual paper-based systems are used whereby the paper with the specifications/instructions gets damaged, misplaced, or switched) can be easily prevented.

To summarize, some of the benefits of using RFID-generated item-level information include (1) refined control of manufacturing systems which leads to quality improvement, (2) timely response in exception handling situations through use of real-time information, (3) the ability to track and trace and to monitor logistics and production operations. As a relatively new technology to wide-spread large-scale manufacturing applications, RFID implementations have potential issues that need to be considered, including (1) high adoption risk since there are only a scattered few ROI evaluations in existence and the complete benefits are somewhat unknown/unmeasurable, (2) the high expense since RFID implementations include the tags themselves in addition to readers, necessary back-end systems, specialized personnel, (3) issues related to security and privacy of the tagged object since it now has locally stored information (unlike bar codes, where information is stored externally in database(s)), and (4) sometimes the potential benefits may not warrant related issues (e.g., resistance) that arise with change.

\section{References}

1. Arkan, I., Landeghem, H.V.: Controlling in-plant Logistics by Deploying RFID System in the Item-level Manufacturing: A Case Study. In: Proceedings of the 13th International Conference on Modern Information Technology in the Innovation Processes of the Industrial Enterprise, MITIP (2011) 
2. Brintrup, A., Ranasinghe, D., McFarlane, D.: RFID Opportunity Analysis for Leaner Manufacturing. International Journal of Production Research 48(9), 27452764 (2010)

3. Higuera, A.G., Montalvo, A.C.: RFID-enhanced Multi-agent Based Control for a Machining System. Int'l J. of Flexible Manufacturing Systems 19(1), 41-61 (2007)

4. Hozak, K., Collier, D.A.: RFID as an Enabler of Improved Manufacturing Performance. Decision Sciences 39(4), 859-881 (2008)

5. Hu, K.F., Zhao, L., Xu, Y.C., Chen, L.: Research on Mining Frequent Path and Prediction Algorithms of Object Movement Patterns in RFID Database. Journal Applied Mechanics and Materials 109, 715-719 (2012)

6. Hua, J., Liang, T., Lei, Z.: Study and Design Real-Time Manufacturing Execution System Based on RFID. In: Proceedings of the Second International Conference on Intelligent Information Technology Application, pp. 591-594 (2008)

7. Huang, G.Q., Zhang, Y.F., Chen, X., Newman, S.T.: RFID-enabled Real-time Wireless Manufacturing for Adaptive Assembly Planning and Control. Journal of Intelligent Manufacturing 19(6), 701-713 (2008)

8. Ivantysynova, L., Klafft, M., Ziekow, H., Günther, O., Kara, S.: RFID in Manufacturing: The Investment Decision. In: Proceedings of the pacific Asia Conference on Information Systems, PACIS (2009)

9. Kapoor, G., Zhou, W., Piramuthu, S.: Challenges Associated with RFID Implementations in Supply Chains. European Journal of Information Systems 18, 526-533 (2009)

10. Kriett, P., Grunow, M., Piramuthu, S.: Knowledge-based Dynamic Job Shop Scheduling with RFID-generated Item-level Information. Working paper (2012)

11. Li, Z., Gadh, R., Prabhu, B.S.: Applications of RFID Technology and Smart Parts in Manufacturing. In: Proceedings of DETC 2004: ASME 2004 Design Engineering Technical Conferences and Computers and Information in Engineering Conference, DETC 2004-57662, p. 7 (2004)

12. Sharp, K.R.: Lessons From The Front: Three Forward-thinking RFID Implementers are Paving the Way for Wide-scale, Real-world Applications. Supply Chain Systems 19(5) (1999), http://209.235.222.4/reader/1999_05/index.htm

13. Thiesse, F., Fleisch, E.: On the value of Location Information to Lot Scheduling in Complex Manufacturing Processes. International Journal of Production Economics 112(2), 532-547 (2008)

14. Trappey, A.J.C., Lu, T.-H., Fu, L.-D.: Development of an Intelligent Agent System for Collaborative Mold Production with RFID Technology. Robotics and Computer-integrated Manufacturing 25(1), 42-56 (2009)

15. Wang, L.-C., Lin, S.-K., Huang, L.-P.: A RFID Based Agile Manufacturing Planning and Control System. In: Wen, P., Li, Y., Polkowski, L., Yao, Y., Tsumoto, S., Wang, G. (eds.) RSKT 2009. LNCS, vol. 5589, pp. 441-451. Springer, Heidelberg (2009)

16. Zhang, Y., Qua, T., Hoa, O.K., Huang, G.Q.: Agent-based Smart Gateway for RFID-enabled Real-time Wireless Manufacturing. International Journal of Production Research 49(5), 1337-1352 (2011)

17. Zhou, W.: RFID and item-level information visibility. European Journal of Operational Research 198(1), 252-258 (2009)

18. Zhou, W., Piramuthu, S.: Manufacturing with Item-Level RFID Information: From Macro to Micro Quality Control. International Journal of Production Economics 135(2), 929-938 (2012)

19. Zhou, W., Piramuthu, S.: Remanufacturing/Refurbishing with RFID-generated Item-Level Information. In: Frick, J., Laugen, B.T. (eds.) APMS 2011. IFIP AICT, vol. 384, pp. 165-170. Springer, Heidelberg (2012) 